\title{
Formulierung und Einhaltung der Ziele
}

Wie bereits in der Einleitung erwähnt, hängt der Erfolg eines Vorhabens von mehreren Faktoren ab. Einer dieser Faktoren ist, das Ziel und die Anforderungen an das Projekt zu kennen. Kennen heißt, eine Abfrage zusammenzustellen und dies schriftlich zu fixieren. Zu dieser Zusammenstellung kann je nach Notwendigkeit interdisziplinäre Mitglieder hinzugezogen werden. Dieses Ziel sollte zumindest das Management unterschreiben. Auf unser Thema „Sanieren im Bestand oder Neubau" angewendet, ergeben sich folgende Fragen (s. Abschn. 2.2, Site-Masterplan):

- Wie wird sich die wirtschaftliche Lage für die Immobilie im Laufe der Zeit ändern (Reparatur und Instandhaltung, Vergrößerung der Familie oder der Produktion u. a.)?

- Was ist der erforderliche Ertrag oder das Produktvolumen?

- Wie viel Platz wird benötigt (Flächenanalyse, Masterplan)?

- In welchem Verhältnis wächst die Infrastruktur mit der Erweiterung oder Verkleinerung der Produktion mit?

- Welche zukünftigen Personalanforderungen bestehen (Privat: Pflege und Betreuung, Industrie: Spinde, Waschplätze, Aufenthaltsräume u. a.)?

Die Antworten auf diese Fragen sollten eindeutige Ziele und Klarheit für zukünftige Schritte bringen. 